$\begin{array}{rr}\text { JUL } 31997 & \text { ANL-HEP-CP-97-49 } \\ \text { OSTI CONF-970564-- }\end{array}$

\title{
The Fermilab Long-Baseline Neutrino program
}

\author{
Maury Goodman \\ for the MINOS collaboration
}

HEP362, Argonne National Lab, Argonne IIl. 60439, USA

\begin{abstract}
Fermilab is embarking upon a neutrino oscillation program which includes a long-baseline neutrino experiment MINOS. MINOS will be a 10 kiloton detector located $730 \mathrm{~km}$ Northwest of Fermilab in the Soudan underground laboratory. It will be sensitive to neutrino oscillations with parameters above $\Delta m^{2} \sim 3 \times 10^{-3} e V^{2}$ and $\sin ^{2}(2 \theta) \sim 0.02$.
\end{abstract}

\section{INTRODUCTION}

The new neutrino beam from the Fermilab Main Injector is known as the NuMI program. There will be a short-baseline experiment, COSMOS based on an emulsion target, and a long-baseline experiment, MINOS which will include a new 10 kiloton detector and the existing 1 kiloton Soudan 2 detector. Here I will describe some of the considerations in the design of long-baseline neutrino oscillation experiments, and the physics goals and status of the design of the new MINOS detector.

DIGTRIBUTION OF THIS DOCUMENT IS UNUWHED

\section{DESIGN OF A LONG-BASELINE NEUTRINO EXPERIMENT}

The basic equation for a neutrino oscillation experiment is

$$
P=\sin ^{2} 2 \theta \sin ^{2} 1.27 \Delta m^{2} L / E_{\nu}
$$

The two physics parameters which the experimenter can measure are the mixing angle $\sin ^{2}(2 \theta)$ and the difference between the squares of the two neutrino masses $\Delta m^{2}$. In order to do this, one designs a test which is sensitive to the presence or absence of neutrino oscillations, yielding $\langle P\rangle$ in the presence of oscillations, or the lowest possible $P_{\min }$ in the absence of evidence for oscillations. The distance between the neutrino source and the experiment

Conference paper submitted to the proceedings of the Sixth Conference on the Intersections of Particle and Nuclear Physics, May 27 - June 2, 1997.
The submitted manuscript hes been authored by a contrector of the U.S. Government andract Na. W-3t-109ENG-38. Accordingly, the U.S. Government retwits a

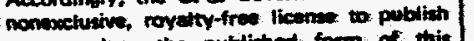

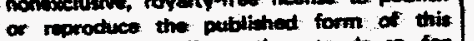
corntrodion, of alfow othen to do $\infty$, for Contriburion, of alfow othe 


\section{DISCLAIMER}

This report was prepared as an account of work sponsored by an agency of the United States Government. Neither the United States Government nor any agency thereof, nor any of their employees, makes any warranty, express or implied, or assumes any legal liability or responsibility for the accuracy, completeness, or usefulness of any information, apparatus, product, or process disclosed, or represents that its use would not infringe privately owned rights. Reference herein to any specific commercial product, process, or service by trade name, trademark, manufacturer, or otherwise does not necessarily constitute or imply its endorsement, recommendation, or favoring by the United States Government or any agency thereof. The views and opinions of authors expressed herein do not necessarily state or reflect those of the United States Government or any agency thereof. 


\section{DIsclamiar}

Portions of this dosoment may be illegible in electronic image produets. Images are produced from the best available original documenter 
$\mathrm{L}$, and the neutrino spectrum with an average energy $\left\langle E_{\nu}\right\rangle$ are basic design parameters of the experiment. Other important factors are the mass of the detector $M$ and properties of the detector such as its energy resolution and spatial granularity.

It is useful to see how the sensitivity in parameter space changes as $L$ and $\mathrm{E}$ are varied. This can be easily studied with the approximation shown in Figure 1. At high $\Delta m^{2}$ the effect of the oscillation washes out the effect of the energy spectrum and $P_{\min }=\frac{1}{2} \sin ^{2}(2 \theta)$. At low $\Delta m^{2}$ we use the fact that

$$
\left.\frac{\partial \Delta m^{2}}{\partial \sin ^{2}(2 \theta)}\right)_{\sin ^{2}(2 \theta)=1}=-\frac{1}{2}
$$

so the slope on a $\log \left[\sin ^{2}(2 \theta)\right]-\log \left[\Delta m^{2}\right]$ plot at low $\Delta m^{2}$ is $-1 / 2$. We can use these two-line limit plots to study scaling effects.

The $\mathrm{L}$ dependence has no clear optimum. As shown in Figure 2, as a fixed size detector is moved further from the $\nu$ source, the sensitivity in $\Delta m^{2}$ increases for a statistical test. (Note that this result does not apply to a background-free test.) However, due to the smaller number of events, the sensitivity to $\sin ^{2}(2 \theta)$ decreases. Thus the placement in $L$ requires a value judgement concerning the region of neutrino oscillation parameter space to explore.

The energy dependence is more complicated. The parameter under primary control is not the neutrino energy, but the accelerator or proton energy used

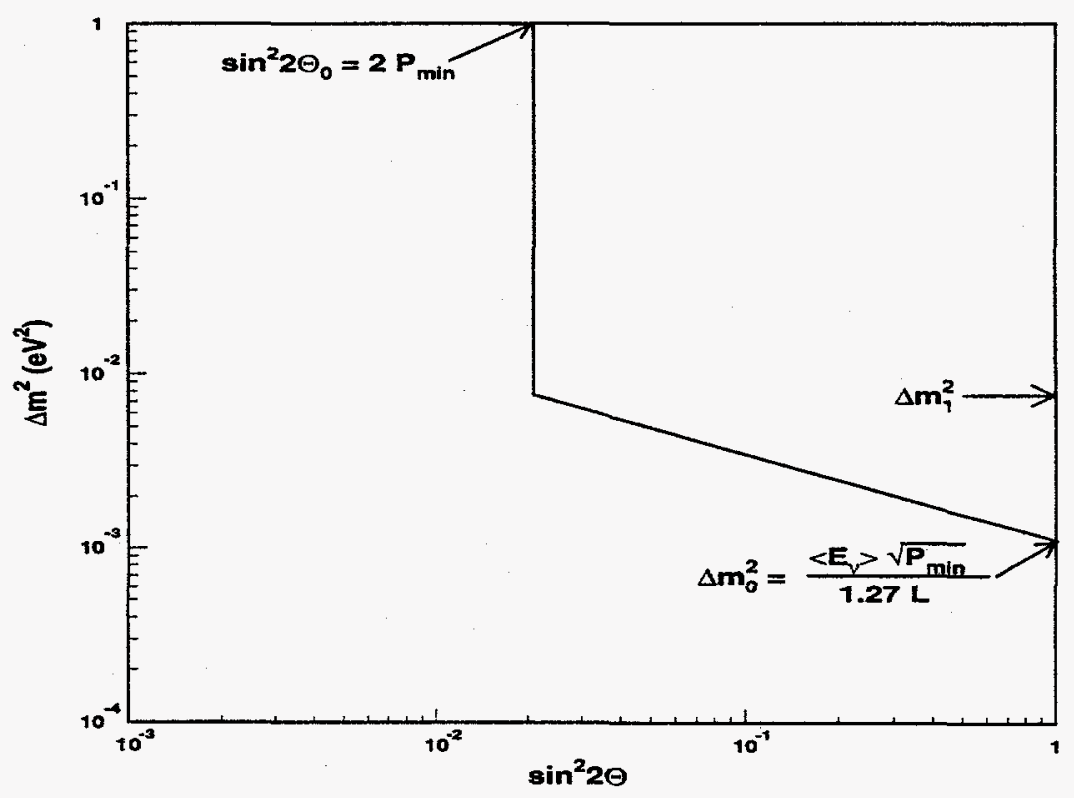

FIGURE 1. Approximation for a neutrino oscillation limit curve showing the relationship between $P_{\min }$ and the limits at high and low $\Delta m^{2}$. 


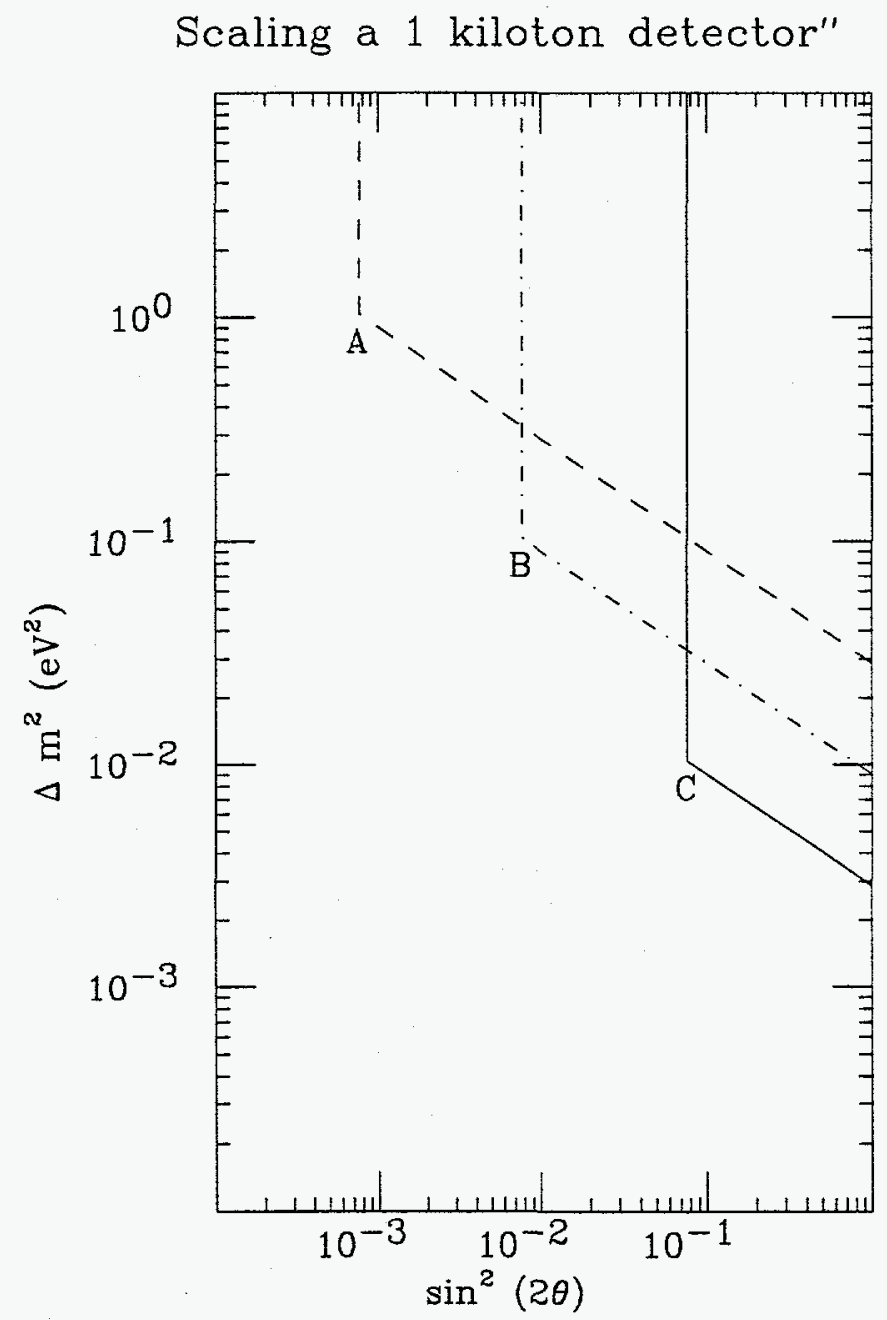

FIGURE 2. Effect of moving a 1 kiloton detector from $7 \mathrm{~km}$ (" $A$ ") to $73 \mathrm{~km}$ ("B") to $730 \mathrm{~km}$ ("C") from the Fermilab NuMI beam. The pattern of these curves will be followed by any test in which $P_{\min } \propto N^{-1 / 2}$. 
to create the neutrino beam. The average neutrino energy only increases logarithmically with $E_{\mathrm{p}}$. There are four advantages to higher proton energy: 1) There are more $\pi$ 's and K's per proton, 2) The $\nu$ beam is more forward, 3) The $\nu$ cross section increases with $E_{\nu}$, and 4) The $\nu_{\tau}$ cross section relative to the $\nu_{\mu}$ cross section rises with energy. There are also 3 advantages to lower proton energy: 1) There can be a larger proton current, 2) There are higher probability for $\pi$ and $K$ decays in a fixed length beam pipe and 3) The sensitivity in $\Delta m^{2} \propto E_{\nu}$. To take advantage of $\nu_{\tau}$ interactions, one wants $\left\langle E_{\nu}\right\rangle \gg 4 \mathrm{GeV}$ which is $\nu_{\tau} \mathrm{CC}$ threshold. Above $4 \mathrm{GeV}$, the ratio of the $\nu_{\tau}$ to $\nu_{\mu}$ cross section only increases slowly. A quantitative study for the NC/CC test of $\Delta m_{\min }^{2}$ and $\sin ^{2}(2 \theta)_{\min }$ for $\nu_{\mu} \rightarrow \nu_{\tau}$ gives a fairly flat response from 100 to $400 \mathrm{GeV}$ proton energy. [2]

A powerful test for $\nu_{\mu} \rightarrow \nu_{\tau}$ oscillations is the measure of the apparent $R$ $=\mathrm{NC} / \mathrm{CC}$ ratio in a $\nu_{\mu}$ beam. In the presence of oscillations, the number of $\mathrm{NC}$ and $\mathrm{CC}$ events are:

$$
n_{\text {"cc" }}=\frac{N(1-P+\eta B P)}{1+R} \text { and } n \text { "nc" }=\frac{N(R+\eta(1-B) P)}{1+R}
$$

where $\mathrm{B}=0.17$ is the branching fraction for $\tau^{-} \rightarrow \mu^{-} X, \mathrm{~N}$ is the number of events, $P$ is the oscillation probability (suitably averaged) and $\eta$ is the ratio of the $\nu_{\tau}$ charged current cross section to the $\nu_{\mu}$ charged current cross section. For the Main Injector energy spectrum, $\eta=0.31$. The notation " $c c$ " distinguishes events classified as charged current due to the presence of a muon in the final state from the actual charged current events, which for an incoming $\nu_{e}$ or $\nu_{\tau}$ would be incorrectly classified as nc events. We can use equations 3 to calculate the modified $R$ in the presence of oscillations.

$$
R_{\text {"nc" / "cc" }}=\frac{n \text { "nc" }}{n \text { "cc" }}=\frac{R+\eta(1-B) P}{1-P+\eta B P}
$$

This can be solved for $\mathrm{P}$, and we use $\delta_{R}=R_{\text {obs }}-$ Rtrue:

$$
P=\frac{\delta_{R}}{\eta(1-B)+R(1-B \eta)}
$$

This equation can be used to set a limit on $\mathrm{P}$ in the absence of oscillations by noting that $\sigma_{R} / R=R(1+R) / \sqrt{N R}$ and that $\delta_{R}=1.29 \sigma_{R}$ for a $90 \% \mathrm{CL}$ upper limit for a one-sided Gaussian. Plugging in the values of $R, B$ and $\eta$, we obtain $P_{\min }=1.83 / \sqrt{N}$, where $\mathrm{N}$ is the total number of $\mathrm{nc}+$ cc events expected.

As noted above, choosing the length of the baseline requires a physics value judgment. Such a value judgment was provided by the Fermilab PAC in June 1993 when they requested that a $\nu_{\mu} \rightarrow \nu_{\tau}$ experiment be sensitive to $\Delta m^{2} \sim$ $10^{-2} \mathrm{eV}^{2}$ and $\sin ^{2}(2 \theta) \sim 10^{-2}$. [3] Motivation for this choice of parameters comes from a desire to cover Cabbibo angle-like mixing for a wide range of 
$\Delta m^{2}$, while covering the whole range of $\Delta m^{2}$ for the atmospheric anomaly at high $\sin ^{2}(2 \theta)$. These parameters lead to a requirement $P_{\min } \sim 0.005$. For the Main Injector beam with $\left\langle E_{\nu}\right\rangle=15 \mathrm{GeV}$ this leads to $\mathrm{L}=830 \mathrm{~km}$. The existing Soudan site at $730 \mathrm{~km}$ is an excellent match to this requirement. The requirement on $N$ is 140,000 events. The latest design of the NuMI beam provides 3150 charged current events per kiloton per year at the far detector. We then solve for $\mathrm{M}(\mathrm{kT})$ in the equation

$$
N=140000=1.31\left(\frac{\text { total events }}{C C \text { event }}\right) \times 3150\left(\frac{C C}{k T \text { year }}\right) * 4 \text { years } * A * M
$$

where $\mathrm{A} \sim 0.75$ is the acceptance. This implies the need for a $11.3 \mathrm{kT}$ detector, close to the $10 \mathrm{kT}$ design of MINOS plus the one kiloton existing Soudan 2 detector.

\section{STATUS OF THE DESIGN OF THE MINOS EXPERIMENT}

The MINOS experiment has been designed to take advantage of the high $\nu$ intensity available from the Fermilab Main Injector. The neutrino beam has been described elsewhere $[4,5]$ The far detector will consist of a new 10 kiloton 8 meter diameter iron toroid with 600 or 1200 active detector planes between $2-4 \mathrm{~cm}$ iron plates Figure 3. There will also be a near MINOS detector of mass about one kiloton.

Three technologies for the active detector choice are currently under consideration: aluminum proportional tubes (APT) patterned after streamer chambers, liquid scintillator counters with fiber readout, and solid scintillator. In the latter two technologies, photodetector costs would be kept down by using multichannel 'pixel' photodetectors. APT's give good spatial resolution with strip readout and are a known technology. Issues include their calorimetric response, manufacturing and cost. Scintillators have good timing resolution and provide good EM calorimetry. Issues are the choice of photodetector, cost, and the light yield. MINOS plans to finalize its detector choice by the end of summer 1997.

Besides the NC/CC test, MINOS has been designed to detect $\nu_{\mu} \rightarrow \nu_{\tau}$ oscillations in a number of independent ways. A list of $\nu_{\mu} \rightarrow \nu_{\tau}$ tests to which the MINOS and Soudan 2 detectors will be sensitive in a wide band beam is given here:

1. Ratio of absolute rates in the near and far detectors.

2. $\frac{N C}{C C}$ ratio

3. $E_{\text {tot }}^{\mathrm{cc}}=E_{\mu}+E_{\text {had }}$

4. $E_{\text {had }}^{n c}$ 
5. $R_{\frac{\mu}{\nu}}=$ Muons from Rock / $\nu$ interactions

6. Rate of stopping rock muons

7. Electron identification

8. Quasielastic $\nu_{\tau}$ with $\tau \rightarrow \mu \nu \nu$ events

9. Missing $p_{t}$ in $\tau \rightarrow$ hadrons

10. $\tau \rightarrow \pi$ events

There are also a number of additional physics issues which the experiment plans to address. MINOS can search for $\nu_{\mu} \rightarrow \nu_{e}$ oscillations using several of the above tests. The experiment can also test the Harrison-Perkins-Scott scenario [6] in which both $\nu_{\mu} \rightarrow \nu_{\tau}$ and $\nu_{\mu} \rightarrow \nu_{e}$ oscillations would be detected. There are other signatures for $\nu_{\mu} \rightarrow \nu_{\tau}$ and $\nu_{\mu} \rightarrow \nu_{e}$ in a Narrow Band Beam. A decrease in the absolute rate of events and the $C C$ energy distribution in the absence of a signal in the other tests would be evidence for $\nu_{\mu} \rightarrow \nu_{\text {sterile }}$ oscillations. Differences between $\nu_{\mu}$ and $\bar{\nu}_{\mu}$ signals can be used to search for CP violation and matter enhanced $\nu_{\mu} \rightarrow \nu_{e}$ oscillations. MINOS can also search for gravitationally induced $\nu$ oscillations and look for anything in the beam which could lead to higher than expected event rates in the far detector. MINOS can also study the highest energy atmospheric neutrinos and will be the first large underground magnetic detector for cosmic ray studies.

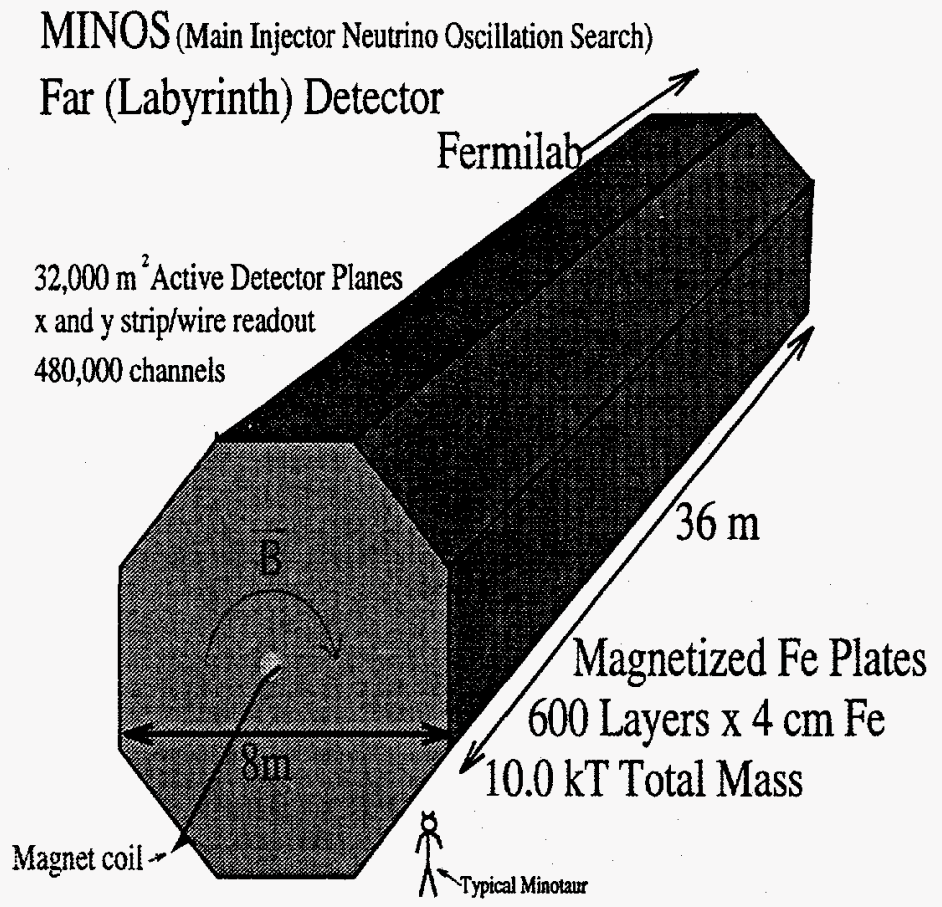

FIGURE 3. The MINOS far detector schematic. 


\section{OUTLOOK}

The Fermilab NuMI beam, the Soudan site, the existing fine-grained Soudan 2 detector, and the planned new $10 \mathrm{kT}$ MINOS detector are well matched to the needs of an ambitious new neutrino oscillation program from the Fermilab Main Injector. MINOS will start taking data whenever the NuMI beam comes on. The present best estimate is 2001.

Further information about the MINOS experiment can be found on the web at http://www.hep.anl.gov/ndk/hypertext/numi.html. A web page devoted to all experiments which study neutrino oscillations is called the "Neutrino Oscillation Industry" and can be found at http://www.hep.anl.gov/ndk/hypertext/nu_industry.html. A monthly email newsletter is also available by sending email to mcg@hep.anl.gov. To get the newsletter, send a message "subscribe". To get a one-line notice when the latest issue has been posted on the web, send "subscribe web".

\section{REFERENCES}

1. W.W.M. Allison et al., NIM A376, 36 (1996).

2. Crane and Goodman, p. 226 in "Particle and Nuclear Astrophysics and Cosmology in the Next Millenium", proceedings of the 1994 Snowmass Summer Study, World Scientific, Kolb and Peccei editors.

3. Fermilab Call for Proposals for a long-baseline neutrino experiment, June 1993.

4. Greg Bock, these proceedings.

5. D. Crane et al., Status Report: Technical Design of Neutrino Beams for the Main Injector, Fermilab-TM-1946, July 1995.

6. P.F. Harrison, D.H. Perkins and W.G. Scott, Phys.Lett. B 349 (1995) 137: B374 (1996) 111; also C. Giunti, C.W. Kim and J.D. Kim, Phys. Lett. B352(1995) 357. 